\title{
The Contemporary Cosmological Models: in Search of the Ways of Systematization
}

\author{
Natalia Semeniuk \\ Doctor of Philosophical Sciences, Associate Professor, National Pedagogical Dragomanov \\ University (Kyiv, Ukraine) \\ E-mail: semenyiuk.nataliya@gmail.com \\ https://orcid.org/0000-0002-6086-0489
}

\begin{abstract}
Modern cosmological models differ in their methodology, tools of argumentation and outlook on the content of the problematic field of study, the apparatus of logical and conceptual justification and the specifics of verification of the results and conclusions. Such pluralism of the epistemological approaches of modern cosmology requires not only ordering but also proper analysis. The article is devoted to the attempt to systematize the main methodological approaches of modern cosmology, and includes the analysis of world-view, philosophical, theological and natural-scientific models of description of modern cosmological projects. The results of the study are important in view of the increase of informative facts on a certain topic in the context of civilization development, rapid processes of the development of the Universe and the development of relevant technologies, as well as the lack of comprehensive interpretation and evaluation of intermediate results and prospects for further theoretical exploration and development.
\end{abstract}

Keywords: cosmology, cosmological model, methodology of cosmological theories, systematization, human development

Received: May 25, 2019; accepted: July 21, 2019

Philosophy and Cosmology, Volume 24, 2020: 29-38.

https://doi.org/10.29202/phil-cosm/24/3

\section{Introduction}

Cosmology as a scientific knowledge of the Universe has a long history of its formation and appears relevant at present. At the same time, the desire to encompass and understand the Universe in its totality is both the virtue and the problematization of scientific search within cosmology. That is why the discourse of cosmological research uses the concepts that are difficult to experimentally prove or refute, and which need philosophical reflection in their implementation. In particular, Konstantin S. Khroutski emphasizes the need for the development of a holistic field of philosophical knowledge — philosophical cosmology, and provides the following definition: "Taking up this position, we formulate the following definition: Philosophical Cosmology is the section of philosophy dealing with the comprehension and cognition of the common universal Process of the Earth's life

(C) Semeniuk, Natalia, 2020 
evolution (CEPEL) and, within it, exploiting the wellbeing of Homo sapience cosmicus the determining unit of this evolutionary Process" (Khroutski, 2001: 208). In his view, in today's society there is a steady demand for such research and knowledge. Being grounded on the traditions of Russian cosmism and the theory of active evolution, he conceptualizes the subject, object, and method of this discipline, its problematic field, and basic theoretical models. The main strategy of this knowledge is named by the author as "comprehensive synthesis", which is demanded by the ideology of "Cosmology Enlightenment".

Complex dialectics of natural scientific and philosophical knowledge in modern cosmological studies is reflected in a purely pragmatic plane. For example, John E. Renesch argues for the importance of world-view transformation in understanding business leadership through the lens of cosmological knowledge, awareness of the complex processes of Human-Universe interaction (Renesch, 1998). The technological age is being replaced with establishment of an era of consciousness, for which the paradigm shifts, complexity and integrity of thinking are not problematic.

Indeed, natural or cosmological activities must be at the heart of social and civilizational advance and development. That is, planetary thinking as a consequence of globalization processes and acceleration of technical impact on the environment requires qualitatively new management, which would be focused on the conservation of the planet's resources and the peaceful exploration of space. It is clear that this movement in theoretical terms should be based on understanding the current trends in the field of cosmology, and precisely the ordering of ideas about modern cosmological models, which our research is aimed at. At the same time, we are aware that there are interesting practical studies in cosmological discourse, such as space law research (Drozd, 2019; Rybachok, 2018), international politics (Svyrydenko \& Yatsenko, 2018), etc., but they are not fundamental and their systematization is not within the scope of this article. Articles in these and other fields seek to address specific problems associated with the processes of space research and exploration, however, cannot be considered cosmological without being relevant to the subject of this field of modern science.

We do not neglect the fact that a number of national and international organizations are trying to integrate cosmological research into their activities. One such example is the activity of the International Society of Philosophy and Cosmology, which has consolidated the research efforts of international experts in philosophy and cosmology from 25 countries for fifteen years (Svyrydenko \& Khomenko, 2019).

\section{Cosmological Issues in the Theological Projection}

Traditionally, cosmological issues were formed in mythological and religious concepts. Questions about the structure and evolution of the Universe, the understanding of space and time, the place and role of consciousness and spirit in the ontology system have consistently been the main content of religious doctrines and myth-making. Modern cosmological studies are also often focused on finding and discovering the potential of religion, Christian in particular, for the basic laws of the Universe. Honorary Professor of Astronomy and History of Science at Harvard University and Honorary Senior Astronomer of the Smithsonian Astrophysical Observatory Owen Gingerich analyzes the transformation of Christian cosmology and theology into modern cosmological studies and proves that Kepler's cosmology is based on metaphorical three-metamorphic symbols, which is not contrary to the Aristotle's geocentric model of the Universe (Gingerich, 2011). 
Professor of Mathematics and Astrophysics Rodney D. Holder updates the cosmological issues that are important in terms of theology: the beginning of the Universe and the essence of the fine-tuning of the Universe for life. According to the author, classical science is not able to answer the fundamental question about the cause of the emergence of the Universe: "It seems to me that modern cosmology poses two fundamental issues that take us beyond the science into the realm of metaphysics and, I shall argue, theology. The first relates to the question of the beginning and to the traditional cosmological argument for the existence of God, in one form or another. The second relates to the so-called fine tuning — the way in which the universe, and the laws of nature describing its structure and evolution, seem to be set up in a very special way in order for intelligent life to evolve at some stage in its history" (Holder, 2016: 234). The author considers the presence of the Absolute to be the most expedient explanation of the Universe: "In addition, design by God is a simple explanation, and much more economical than the multiverse. One is not invoking a whole multitude of complex entities with which one can have no possible interaction, but one intelligent being — like ourselves in some ways, but so much greater: a being of unlimited power and knowledge (omnipotent and omniscient in classical terms), and perfectly good" (Holder, 2016: 253). Accordingly, the concept of the Absolute remains a powerful argument in explaining the diversity of the world and its systematic functioning.

Patrick J. Connolly resuscitates the philosophical interest in the work of Susan Newcome, "Evidence of the Christian Religion," where the author reconstructs the innovative argument about the existence of God: "Specifically, Newcome encourages a richer understanding of causal chains in nature and what is required for their existence. By focusing on the ways in which causal processes rely on external support, Newcome hopes to show that the universe cannot be explained without appeal to a transcendent God working to sustain observed phenomena" (Connolly, 2019: 17). The main essence of this argument is to appeal to Newtonian natural philosophy, which assumes the presence of entropy in the Universe. Accordingly, causal relationships in nature cannot exist autonomously, so they require an external, in relation to the Universe, leader. The authors call this external motive and stabilizing power as God.

This opinion is shared by Wesley J Wildman. He analyzes the relationship between physical and philosophical cosmology, which is to limit the speculative capabilities of philosophical analytics: "In the theistic version of this supernatural cosmology, one omnipotent deity with determinate features is the ultimate reality who creates everything and interacts with the world according to divine purposes. The natural world may or may not reflect the deity's determinate character, just as the potter's clay may be forged into shapes that both express and fail to express the potter's personality. However, the deity is nonetheless responsible for the created world in some ultimate sense. This gives supernatural theism considerable flexibility of interpretation in relation to the metaphysical implications of physical cosmology" (Wildman, 2008: 199). The author notes that these restrictions are not categorical, but have a significant effect on the content of philosophical concepts of cosmology. He distinguishes three different classes of philosophical cosmology: supernatural, procedural, and cosmology of the basis of existence. They all contain original research methodology and design different cosmology models that sometimes share common predications (Wildman, 2008).

Joseph Milne pertinently claims that the obtaining of medieval Christian cosmology, along with Christian mysticism, have a significant influence on modern materialistic cosmology (Milne, 2011). Therefore, the author focuses on the discrepancy between the "inner" aspect of mystical experience and the "outer" conception of nature and the Universe. Accordingly, 
the attempts to reconcile medieval mysticism with modern scientific cosmological concepts are doomed. The author insists on the criticality of judgments in space exploration and the need to abandon stereotypical and unfounded assumptions.

\section{Demarcation methodology in modern cosmology}

In the methodological complex of modern cosmology, the physical, mathematical and philosophical apparatus of the research closely interact. At the same time, the priority of one of them in modern cosmological research is extremely difficult to be specified unequivocally. John E. Renesch regards cosmology as a field of contradiction, and the range of its assessment varies from the fundamental refusal of cosmology in scientific status (its recognition as 'pure fantasy') to the belief in the undeniable value of such research: "Cosmology is a field riddled with controversy. With regard to cosmology itself, there are several different schools of thought. Some think that there is basically no such thing as scientific cosmology, and that cosmology is just a pseudoscience. Alternatively, as the French physicist Brillouin put it, cosmology is 'pure fantasy'. A second school says that the value of cosmology is undeniable. Astronomical observations and measurements have already encountered large-scale problems, and these problems have an objective existence; they are not fabricated. This school holds, however, that because at present observational results are still scant, we should therefore emphasize the accumulation of observations and the organization of data and speculate less about theories or models of the universe. Some observational astronomers hold this view. Then there are still other people who believe that we want not only to emphasize observational facts, but also to make a serious effort to analyze the underlying mechanisms. We should look not only at the universe of the present, but also explore cosmic evolution, developing our cosmology based on the mutual interplay of theory and observation" (Renesch, 1988: 55). Emphasizing the importance of observations in the study and considering their impossibility, the question arises as to the criterion of truth and the possibility of predicting the further unfolding of space evolution.

Thomas Lepeltier, who criticizes Edward Milne's cosmological concept, mentions this very problem. The author considers it to be general and non-observational: "Milne was without doubt a brilliant astrophysicist, and his cosmological model, at the time it was invented, was recognized as original and sophisticated. Having fallen into oblivion since, its rehabilitation by Gale and co. is welcome. However, as the quotations above show, these authors describe Milne as the victorious champion of the hypothetico-deductive method and of rationalism in physics" (Lepeltier, 2006: 473). The great resonance, caused by this work, did not subsequently have a significant impact on cosmological studies, so the author concludes that Milne's rationalism did not change the general methodology of modern cosmological research.

The necessity and impossibility of empirical studies in cosmology are actualized by Martin Rees, who relies on the opinion of cosmologists about the empirical status of cosmological science. The prerequisite for this possibility is a high level of overall symmetry and uniformity. Comparing the methodology of cosmology and biology, the author concludes with the complexity of the problem field, both at macro-level and micro-level, and the level of organic life (Rees, 1999).

Therefore, the problematic field of modern cosmology is a complex of mystical-religious, metaphysical-philosophical, and natural-scientific methods of research and analytics. Judging from these views, Helge Kragh draws attention to the difference between cosmology and other fields of science. The juxtaposition of the standard and speculative models of the 
Universe, the emergence of the idea of the multiplicity of universes (the Multiverse) and the anthropic principle broadens the problematic field of cosmological research. The author tries to discretionarily systematize the theories available in cosmological discourse as follows: "There are several ways in which to classify multiverse theories, but none that has won general recognition. Nor is there, for that matter, consensus as to what constitutes or defines a multiverse. According to Laura Mersini-Houghton it is completely theory dependent, namely 'the ensemble of all possible universes predicted by the underlying theory'. A simple classification has been proposed by George Gale, who distinguishes between (1) spatial multiverse models, (2) temporal multiverse models, and (3) models with universes of other dimensions. The second class comprises cyclic models, new versions of which have recently attracted considerable attention. In some of these models the current and previous cycles are physically unconnected in the sense that no information from the previous universe passes over to the successor universe; in other models the new universe has some memory of its predecessor" (Kragh, 2009: 539). Based on the above, it can be argued that in the current research situation of cosmological discourse, epistemological discussions on the methodology of exploration and substantiation of knowledge about space are of particular importance.

Accordingly, the idea of certain unitarity of research practices and beliefs within established scientific fields will be illusory. For example, Yurij Baryshev conceptualizes the main content of the problems of modern cosmological studies, among which the most important ones are the expansion of space, the comparison of the rate of decline of distant galaxies with the speed of light, the problem of gravity and cosmological constants. However, there is no clear answer to any of these questions, but only a field of heated debate and competing hypotheses (Baryshev, 2000).

The problem of time and space is the main one in modern cosmology. Logically, the existence of time beyond space is impossible. However, cosmological theories project the ideas about the space of possible orders that contain primal temporal dynamics, or quantumcosmological models, exploring the function of the waves of the Universe and claiming its timeless nature. Therefore, time is proclaimed to be the effect of perception of the macrocosm. In addition, different modes of temporality are possible in the complex hierarchy of reality, so the only time for all astrophysical objects is not only difficult to imagine but also unnecessary. Equally important to modern cosmology is the notion of the Multiverse, a complex model of interaction and the mutual existence of the n-number of Universes. Changing the status of this object from a hypothetical to a real can be enabled by studying the astrophysical phenomenon called a 'mole hole', which, according to researchers, not only combine different Universes, but also allows the circulation of matter and information throughout a complex structure of being. Consequently, if the beliefs about the model and patterns of being in the Universe do not prevail, they definitely extend the boundaries of our vision.

The subjectivity of ideas is shaped by culture and tradition. Therefore, these are questions of morale and morality, which, according to the purpose of our study, can have a corresponding impact on the nature and content of cosmological theories. Ed Luker looks at the problem of moral imperative from a cosmological perspective. Analyzing works of art and their historical context, the author proves the problematic justification of moral imperative in the conditions of technological progress: "It is not just our attentive capacities that are held by the dominating force of this meniscus but also the landscape we were previously asked to attend to. In contrast to the emphasis on nature and place in The White Stones, one might consider this poem to enact a 'metabolic rift' between man and nature, understood poetically. The 'metabolic rift' is a concept developed by Marx in Capital vol. III. It identifies that nature 
becomes an object entirely appropriated and reigned to the needs of capital by human labour" (Luker, 2016: 15). This is literature that is regarded as a valuable asset in understanding, interpreting, and furthering cosmological research.

The researchers from James Douglas Orton and Kari A. O'Grady perform a qualitative meta-analysis of the concept of 'cosmology episode'. The authors consider literature to be the first finding, in which the episodes of cosmology are mentioned at the individual, team, organizational, social and national level. The second finding is that cosmological episodes have a procedural character of stability. The third one is typologizing literature into catastrophic, contextual, ancillary and metaphorical episodes. The authors summarize: "Contextualized cosmology episodes impact people within a specific high-risk industry context - in contrast to catastrophic cosmology episodes with their impact on entire nations, and disastrous cosmology episodes with their impact on specific geographic regions" (Orton \& O’Grady, 2016: 14).

Therefore, as modern cosmologists point out, there is no longer a demarcation line between physics and metaphysics in the twentieth century. Physical-mathematical models of the reality of modern cosmology significantly change the perception of order and causality. Yet, ordering is defined not only as the geometric time that took place in the classic model of the Universe. Geometric time means marking objects of reality into a certain established system of mutual coherence. Non-classical models produce a different notion of order, in which the factor of systematization is not a spatial-temporal localization, but an expedient conceptual content.

\section{Pluralism of modern cosmological models}

Therefore, the content of modern cosmological queries is to find out the possible answers to the following questions:

1. About the nature of reality (reality as an object of observation and / or speculative object of philosophical reflection).

2. About the nature of cognition (interaction of theoretical and experimental research methodology).

3. About the nature of human activity (the question of the anthropic principle: reality is static or plastic under the presence of consciousness).

Modern cosmological studies offer different models of answers to these fundamental queries. Oleg Bazaluk and Iliana Vladlenova provide the following definition: "Cosmological models are physical-mathematical models that seek to describe the development of the Universe as a whole. The use of modelling in cosmology is due to the complexity of processes and phenomena occurring in the Universe, as well as the increase in the pace of mathematization and the expansion of its scope. Thus, the cosmological model is a substitute object of the original object, which provides the study of some qualities of the original and gives information about its most important qualities" (Bazaluk \& Vladlenova, 2013: 51). The most traditional, according to the authors, is a mechanistic model of the Universe, namely "Newton's cosmological model is based on the achievements of classical mechanics of the $17^{\text {th }}-18^{\text {th }}$ centuries and is, therefore, of a purely mechanistic nature, reflecting the way of explaining the motion and interaction of the objects of study based on mechanical regularities. The Newtonian model of the Universe contained three basic tenets: stationarity, the invariability of the Universe in time; homogeneity and isotropy; lack of privileged directions; Euclidean space" (Bazaluk \& Vladlenova, 2013: 62). This model reflects the 
basic principles of classical metaphysics and the corresponding categorical apparatus of the research.

Modern cosmological models also often imply determination, causality, time and space in other worldviews. Robert L. Oldershaw proposes a cosmological model called 'self-similar hierarchical cosmology', according to which nature is regarded as organized into a transfinite hierarchical structure of self-similar systems: "Local hierarchical organizations are defined as those that are a function of spatial or temporal considerations, whereas global hierarchical organization is independent of location and time. Our current cosmological paradigm, based on the various types of 'Big Bang' models that involve an initial cosmological singularity, treats nature's hierarchical organization as being independent of spatial considerations, as expressed in the Cosmological Principle, but very much a function of time. The hierarchical organization of nature is interpreted within this paradigm has having resulted from a sequence of 'condensations' of matter occurring after the 'Big Bang'. According to this paradigm the universe begins and ends without hierarchical organization; multi-levelledness is a function of time and therefore the 'Big Bang' paradigm does not involve global hierarchical organization. The self-similar hierarchical paradigm, on the other hand, views nature's hierarchical organization as being neither a function of spatial considerations nor a function of time, hence the Hierarchical Principle which asserts that nature's hierarchy is global" (Oldershaw, 1985: 248). The author understands self-similarity as a discrete rather than a continuous form of conformal invariance. He explains this position by the presence of large intervals between analogue systems on a spatial-temporal scale. It is noted that the identified analogue systems from galactic, stellar and atomic scales will have formally similar morphologies kinematics and dynamics. The author offers the criteria for empirical justification of this model and the mathematical apparatus. The idea of the formation and perspective of the self-similar hierarchical paradigm of the Universe research is developed.

The justification of the anthropic principle, the implementation of which contains a powerful epistemological potential, is important in modern cosmological models. Thus, Milan M. Ćirković (Ćirković, 2004) notes that after classical criticism in modern research, the place of the anthropic principle in distinguishing cosmological models should be revised: "The simplest way to divide all cosmologies is into two broad classes: those postulating the eternal universe and those which postulate some origin of the Universe, or at least the part of it that cosmologists are currently inhabiting. Eternal universes (and here by eternal I mean those with no beginning or end, or even only those with no beginning) are the only ones that could pretend to adopt some sort of stationarity, a condition which is of singular importance in many branches of physics (among other issues because the law of energy conservation is closely connected with a translational symmetry of time), and which is certainly seen as greatly simplifying the solution of specific problems everywhere" (Ćirković, 2004: 567). The author argues that the anthropic principle is important for further cosmological studies, especially in current inflation models such as the theory of chaotic Linde inflation. In his view, the cognitive value of the anthropic principle is expedient in solving the end-to-end problems of third-party rational observers and in the context of temporal infinity.

Ian G. Moss explores the theoretical implications of the discovery of the Higgs boson, which reveals the possible pluralism of cosmological scenarios and produces the potential to emerge from standard models of particle physics and cosmology (Moss, 2015). A cosmological model of a different kind is offered by Anna Popova, who studies the cosmological solutions of the spatial dimensions of galaxies with respect to their age and chemical composition. In addition, the author proposes her own procedure and classification for determining the 
dimension and other parameters of our Universe: "With these formulations of WAP in mind, it is clear why the Davies' argument against the Ellis et al. cosmological model can be called anthropic at all; it takes into account the restrictions to be imposed on cosmological models following the existence of a specialized subclass of intelligent observers, namely the 'technological communities" (Popova, 1995: 570).

When following the integrative methodology of cosmological research, we find obvious prerequisites for dialectical opposition, which is transformed into an extensive network of negative dialectics. Modern physics offers, together with the classic mechanical model of the Universe, quantum (Max Planck) and relativistic (Albert Einstein), which fundamentally change the concept of the nature of space and time and patterns of interaction. One version of such non-classical concepts is 'pancake theory' by Iakov Zeldovich, one of the most influential researchers in cosmology of the century. This theory analyzes the problem of symmetry and gravity and designs the configurations of space objects: "The growth of fluctuations in both adiabatic and isothermal models are described, and nicely detailed through the use of graphs to make it easier to understand how the form of the fluctuations changes between epochs. A big emphasis is placed on the role of the inflationary universe, on the possibility of a neutrino dominated universe, and the importance of pancake structures for the bubbly structure seen in our universe" (Zeldovich, 1976: 136).

The Big Bang theory, or the theory of the genesis of the Universe of singularity, has gained wide recognition. Modern researchers have criticized the definition of singularity as infinity, and have suggested replacing these quantities with a Planck, that is, a quantum description. Proponents of the Big Bang theory, in order to correlate theoretical and experimental data, work out the so-called 'inflation model', which assumes the existence of the 'pre-singular' state of matter.

A complex hierarchical model of reality is offered by the holographic model of the Universe, according to which reality is a multilayered formation of sub-physical forms of matter, which are various in quality. Similar in content is the fractal model of the Universe, according to which the diversity of reality focuses on a particular configuration of particles, which is repeated in different modifications at different levels of being. Another interpretation of reality is offered by the so-called information models of the world. According to these concepts, the basis of the Universe is not matter, but information, and its movement and transmission. Matter in such a theory plays a supporting role, such as technical equipment. Although these models are more often an object of criticism of modern cosmologists, we cannot avoid them trying to show the breadth of interpretation of cosmological issues.

\section{Conclusions}

As a result of an attempt to propose the author's approaches to the systematization of cosmological models, the following conclusions were reached:

1. Modern cosmology is a science with a long history of research and uncertain status.

2. The range of its estimates varies from the definition of cosmology as a promising and accurate science of physical-mathematical character to its philosophical and theological expediency.

3. The debatable status of cosmology as a science is reflected in its methodology, which demonstrates the realization of the principle of demarcation between natural and philosophical knowledge.

4. The philosophical and theological component of cosmological research not only outlines the worldview paradigm of interpretation and study of the Universe, but also 
provides the necessary justification for explaining the scale of the outer space, which far outweighs the possibilities of human subjectivity.

Scientific cosmological models create new projections of orientation and interpretation of space and time, specifics of dynamic processes of the Universe and formation of strategies of human activity explication according to ontological shifts and universals.

\section{References}

Baryshev, Yurij (2000) Conceptual problems of fractal cosmology. Astronomical \& Astrophysical Transactions, Vol. 19, Issue 3-4, 417-435. https://doi. org/10.1080/10556790008238588

Bazaluk, Oleg, and Iliana Vladlenova (2013). The Philosophical Problems of Cosmology: Monograph. Kharkov: KhPI. (in Russian)

Ćirković, Milan M. (2004) The anthropic principle and the duration of the cosmological past. Astronomical \& Astrophysical Transaction. The Journal of the Eurasian Astronomical Society, Vol. 23, Issue 6, 235-255. https://doi.org/10.1080/10556790412331335327

Connolly, Patrick J. (2019) Susanna Newcome's cosmological argument. British Journal for the History of Philosophy, Vol. 27, Issue 4, 842-859. https://doi.org/10.1080/096087 88.2018.1494543

Contemporary Cosmology: The Philosophical Horizons (2011) V.V. Kazyutinski, editor. Moscow: Kanon+, ROOI "Rehabilitation" (in Russian)

Copeland, Edmund (1992) Modern cosmology. Contemporary Physics, Vol. 33, Issue 2, 135 136. https://doi.org/10.1080/00107519208219538

Drozd, Olexiy (2019) Regulatory Framework for Space Activities: the Analysis of the Main Regulatory Acts. Advanced Space Law, Volume 3, 18-29. https://doi.org/10.29202/ asl $/ 2019 / 3 / 2$

Ebury, Katherine (2014) “A new science": Yeats's A Vision and relativistic cosmology. Irish Studies Review. Some millennial thoughts on cosmology, Vol. 22, Issue 2, 167-183. https://doi.org/10.1080/09670882.2014.891379

Gingerich, Owen (2011) Kepler's Trinitarian Cosmology. Theology and Science, Vol. 9, Issue 1, 235-255. https://doi.org/10.1080/14746700.2011.547004

Holder, Rodney D. (2016) Explaining and Explaining Away in Cosmology and Theology. Theology and Science, Vol. 14, Issue 3, 234-255. https://doi.org/10.1080/14746700. 2016.1191876

Khroutski, Konstantin S. (2001) Introducing philosophical cosmology. World Futures._The Journal of New Paradigm Research, Vol. 57, Issue 3, 201- 212. https://doi.org/10.10 80/02604027.2001.9972829

Kragh, Helge (2009) Contemporary History of Cosmology and the Controversy over the Multiverse. Annals of Science, Vol. 66, Issue 4, 529-551. https://doi. org/10.1080/00033790903047725

Leon, Justin (1999) Lakota experiences of (In)security: cosmology and ontological security. International Feminist Journal of Politics, Vol. 24, Issue 4, 261-265. https://doi.org/1 $0.1080 / 14616742.2018 .1527183$

Lepeltier, Thomas (2006) Edward Milne's influence on modern cosmology. Annals of Science, Vol. 63, Issue 4, 471-481. https://doi.org/10.1080/00033790600718578

Luker, Ed (2019) J. H. Prynne's moral cosmology. Textual Practice, Vol. 33, Issue 7, 1131 1152. https://doi.org/10.1080/0950236X.2017.1417894 
Milne, Joseph (2011) Mystical Aspects of Christian Cosmology. Medieval Mystical Theology, Vol. 20, Issue 1, 47-65. https://doi.org/10.1558/mmt.v20.47

Moss, Ian G. (2015) Higgs boson cosmology. Contemporary Physics, Vol. 56, Issue 4, 468476. https://doi.org/10.1080/00107514.2015.1058543

Oldershaw, Robert L. (1985) Set theory applied to the self-similar hierarchical cosmology. International Journal of General Systems, Vol. 10, Issue 4, 235-255. https://doi. org/10.1080/03081078508934891

Orton, James D., and Kari A. O’Grady (2016) Cosmology episodes: a reconceptualization. Journal of Management, Spirituality \& Religion, Vol. 13, Issue 3, 226-245. https:// doi.org/10.1080/14766086.2016.1159975

Philosophical Problems of Modern Cosmology (1988) Chinese Studies in Philosophy, Vol. 19. Issue 4, 55-64. https://doi.org/10.2753/CSP1097-1467190455

Popova, Anna (1995) Friedmann cosmology in alternative spatial dimensions. Solutions and tests. Astronomical \& Astrophysical Transactions, Vol. 8, Issue 3, 165-194. https:// doi.org/10.1080/10556799508203305

Rees, Martin (1999) Some millennial thoughts on cosmology. Interdisciplinary Science Reviews, Vol. 24. Issue 4, 261-265. https://doi.org/10.1179/030801899678920

Renesch, John E. (1998) New leaders for a new future: The new business cosmology. World Futures. The Journal of New Paradigm Research, Vol. 52, Issue 2: The Evolution of the Culture of the Enterprise: Lessons for and from Hungry, 131-135. https://doi.org/ $10.1080 / 02604027.1998 .9972700$

Rybachok, Victoria (2018) The Term "Space Object" and its Legal Status. Advanced Space Law, Vol. 2, 44-49. https://doi.org/10.29202/as1/2018/2/6

Svyrydenko, Denys, and Hleb Khomenko (2019) International Society of Philosophy and Cosmology: Fifteen-Year History and Modernity. Philosophy and Cosmology, Vol. 23, 178-187. https://doi.org/10.29202/phil-cosm/23/17

Svyrydenko, Denys, and Olena Yatsenko (2018) Dialectics of Nominal and Real Power in the Ukrainian and World Politics. Ukrainian Policymaker, Vol. 2, 33-40. https://doi. org/10.29202/up/2/5

The Controversy Between Science and Pseudoscience over Modern Cosmology (1988) Chinese Studies in Philosophy, Vol. 19. Issue 4, 14-26. https://doi.org/10.2753/ CSP1097-1467190414

Wildman, Wesley J. (2008) The Import of Physical Cosmology for Philosophical Cosmology. Theology and Science, Vol. 6. Issue 2, 197-212. https://doi. org/10.1080/14746700801976940

Zeldovich, Iakov, and Ivan Novikov (1976). Contemporary Trends in Cosmology. Soviet Studies in Philosophy, Vol. 14. Issue 4, 28-49. https://doi.org/10.2753/RSP10611967140428 